\title{
AU1 Harnessing Omics Big Data in Nine Vertebrate Species by Genome-Wide Prioritization of Sequence Variants with the Highest Predicted Deleterious Effect on Protein Function
}

\author{
Vita Rozman and Tanja Kunej
}

\begin{abstract}
Harnessing the genomics big data requires innovation in how we extract and interpret biologically relevant variants. Currently, there is no established catalog of prioritized missense variants associated with deleterious protein function phenotypes. We report in this study, to the best of our knowledge, the first genome-wide prioritization of sequence variants with the most deleterious effect on protein function (potentially deleterious variants [pDelVars]) in nine vertebrate species: human, cattle, horse, sheep, pig, dog, rat, mouse, and zebrafish. The analysis was conducted using the Ensembl/BioMart tool. Genes comprising pDelVars in the highest number of examined species were identified using a Python script. Multiple genomic alignments of the selected genes were built to identify interspecies orthologous potentially deleterious variants, which we defined as the "ortho-pDelVars." Genome-wide prioritization revealed that in humans, $0.12 \%$ of the known variants are predicted to be deleterious. In seven out of nine examined vertebrate species, the genes encoding the multiple PDZ domain crumbs cell polarity complex component (MPDZ) and the transforming acidic coiled-coil containing protein 2 (TACC2) comprise pDelVars. Five interspecies ortho-pDelVars were identified in three genes. These findings offer new ways to harness genomics big data by facilitating the identification of functional polymorphisms in humans and animal models and thus provide a future basis for optimization of protocols for whole genome prioritization of pDelVars and screening of orthologous sequence variants. The approach presented here can inform various postgenomic applications such as personalized medicine and multiomics study of health interventions (iatromics).
\end{abstract}

Keywords: big data, potentially deleterious variants (pDelVars), orthologous potentially deleterious variants (ortho-pDelVars), PolyPhen (polymorphism phenotyping), SIFT (sorts intolerant from tolerant), single-nucleotide polymorphism (SNP)

\section{Introduction}

$\mathbf{H}$ ARNESSING THE GENOMICS BIG DATA requires rethinking how we prioritize the biologically relevant variants generated by high-throughput analyses. In this context, deleterious variants that negatively impact the protein function are often associated with diseases as well as other important traits (Sachidanandam et al., 2001; Xue et al., 2012). Moreover, most mutations in functional sites are expected to be deleterious, with a different severity of fitness effects (Makino et al., 2018).

Genetic disorders vary from simple monogenic (Mendelian disorders), caused mainly by various sequence variants of a single gene, to oligo- and polygenic disorders affected by a few or several genes (Care et al., 2009). Sequence variants comprise single-nucleotide polymorphisms (SNPs), insertions (insertion of one or several nucleotides), deletions (deletion of one or several nucleotides), indels (an insertion and a deletion, affecting two or more nucleotides), and substitutions (a sequence alteration where the length of the change in the variant is the same as that of the reference) (Cunningham et al., 2015). The most abundant of genetic variants in human genomes are SNPs (>99.9\%) followed by indels and structural variants (1000 Genomes Project Consortium et al., 2015). Depending upon how it affects the amino acid, a SNP in the protein coding region can be either synonymous, nonsynonymous (nsSNP), or nonsense (Wong and Zhang, 2014). The nsSNPs that cause amino acid substitutions may affect protein functions in different ways. They may be: (1) beneficial and elicit a selective sweep, (2)

Department of Animal Science, Biotechnical Faculty, University of Ljubljana, Domžale, Slovenia. 
impose a severe fitness cost and may be very rarely sampled, (3) neutral, or (4) neither beneficial nor excessively harmful, but slightly deleterious (Balbi and Feil, 2007).

Some variants are located at the same genomic position or in the same nucleotide triplet (codon) of a gene in genomes of two (or more) species. Such variants that are in the present study referred to as orthologous variants (orthoVars) might be the reason for specific genetic diseases. Identification of orthoVars is of importance since they can cause a similar disease in different species. Chen et al. (2016) conducted a genome-wide analysis of coincident single-nucleotide polymorphisms (coSNPs) of orthologous positions in coding sequences in five primates: human, chimpanzee, orangutan, gorilla, and rhesus macaque. They concluded that the orthoVars of human disease-associated variants might also be associated with the corresponding diseases in the compared species (Chen et al., 2016).

Tools for prediction of potentially deleterious (termed also intolerant or nontolerated) SNPs have already been developed, namely the PolyPhen-2 tool (Adzhubei et al., 2013) and the sorts intolerant from tolerant (SIFT) tool (Kumar et al., 2009), which are publicly accessible in the Ensembl/BioMart tool (Cunningham et al., 2015). SIFT prioritizes potentially deleterious variants (pDelVars) in animals, whereas in human, polymorphism phenotyping (PolyPhen) is available as well. The tools predict whether an nsSNP that causes a single amino acid substitution in a protein sequence affects the protein function.

Despite advances to identify an immense abundance of nsSNPs among humans (Wong and Zhang, 2014) and in other species, there is no established catalog of prioritized missense variants associated with deleterious protein function phenotypes.

We report here, to the best of our knowledge, the first genome-wide prioritization of sequence variants with the most deleterious effect on protein function (i.e., pDelVars) in nine vertebrate species: human, cattle, horse, sheep, pig, dog, rat, mouse, and zebrafish.

Specifically, the aim of the present study was (1) to perform genome-wide screening for pDelVars in nine vertebrate genomes and (2) extract their functional annotation from various databases. Furthermore, we identified genes with the highest number of pDelVars within each individual species and gene(s) containing pDelVars in the highest number of species. The results also revealed interspecies orthologous pDelVars, which we defined as orthopDelVars.

\section{Materials and Methods}

\section{Genome-wide screening for pDelVars}

In silico genome-wide screening for pDelVars was performed in nine species: human and eight animal genomes: zebrafish, mouse, rat, dog, pig, sheep, horse, and cattle. pDelVars were prioritized using the BioMart data-mining tool by Ensembl, release 85 (www.ensembl.org/biomart). Information regarding the reference genomes implemented in

T1 the present study can be found in Table 1 .

The main species of interest for prioritization of pDelVars and further functional characterization was human, however, seven additional species of mammals (mouse, rat, dog, pig, sheep, horse, and cattle) and a vertebrate model organism

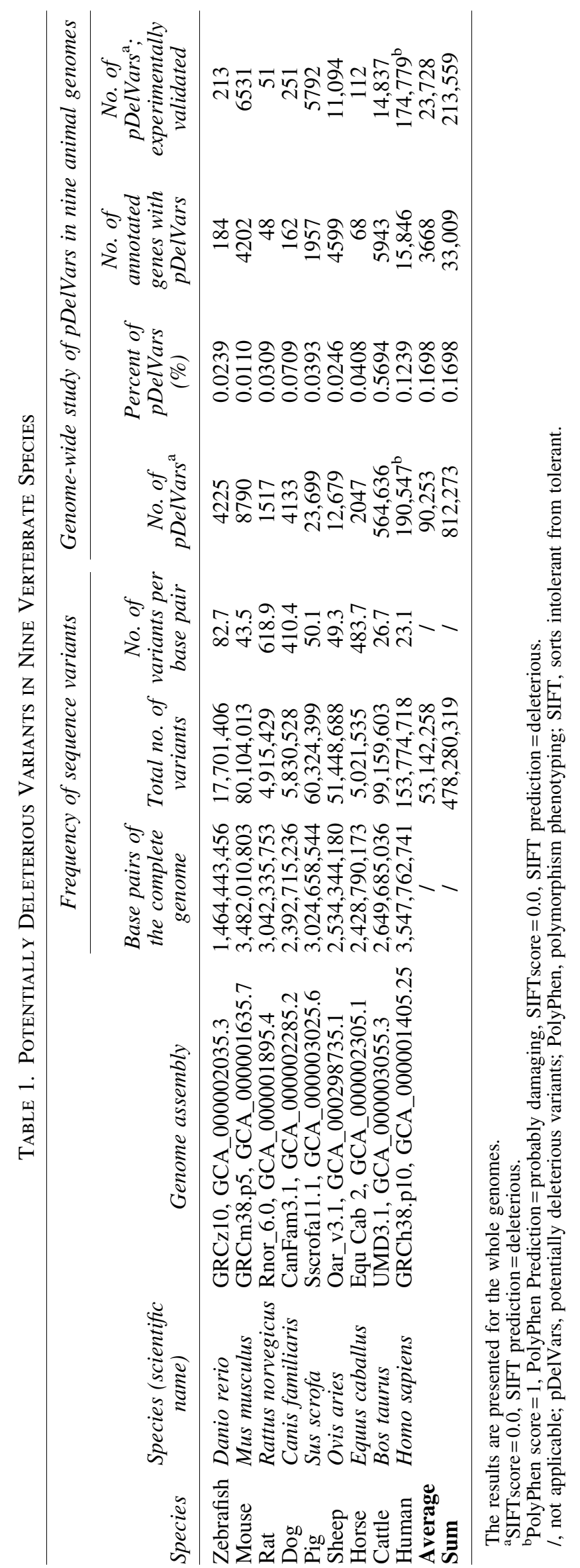


(zebrafish) were included as well to compare the data on pDelVars and identify interspecies ortho-pDelVars.

Prioritization of pDelVars was conducted in each individual species separately using "short variants" option in BioMart. Variants were prioritized using the SIFT tool (Kumar et al., 2009) that determines the SIFT score and predicts whether an nsSNPs is deleterious (0.0) or tolerated (1.0), based on normalized probabilities for all possible 20 amino acids at that position (Kumar et al., 2009; Ng and Henikoff, 2001). However, in human the PolyPhen (Adzhubei et al., 2013) tool for prediction of the possible impact of an amino acid substitution on the structure and function of a human protein is available as well. An nsSNP is qualitatively categorized as benign, possibly damaging, or probably damaging along with a numerical score ranging from 0.0 (benign) to 1.0 (damaging), based on the calculation of a Naïve Bayes posterior probability (Adzhubei et al., 2010, 2013).

In zebrafish, mouse, rat, dog, pig, sheep, horse, and cattle genome-wide filtering for nsSNPs with the most deleterious effect on a protein function was applied using SIFT (SIFT score $=0.0$ ). Additional filter "variant supporting evidence only" was selected to obtain pDelVars, which are experimentally validated.

Due to an immense amount of genomic data in human, genome-wide filtering is not possible. Therefore, screening of the individual chromosomes was performed and then the data were merged. The nsSNPs with the most deleterious effect on protein function in human were obtained using both the SIFT (score $=0.0)$ and the PolyPhen $($ score $=1.0)$ tool. The following attributes were selected: variant name, variant source, chromosome name, chromosome position start (base pair [bp]), chromosome position end (bp), and Ensembl gene ID. In addition, gene names, gene descriptions, and associated phenotypes were extracted using Ensembl/BioMart tool/ Ensembl Genes (www.ensembl.org/biomart/martview), where a list of Ensembl IDs for each species was uploaded and filtered for the desired attributes.

Genes with the highest number of pDelVars within each individual species and genes comprising pDelVars in at least seven out of nine species were identified using a custom Python script, written in the Python programming language (Python Software Foundation. Python Language Reference, version 3.5., www.python.org). The script counts the frequencies of the words in the input list of gene names (.txt format). Genes with the highest number of pDelVars were determined in each individual species separately and for all animal species together. Similarly, the same script was used to identify the genes comprising pDelVars in the highest number (at least seven out of nine) of examined species, where the input file was a list of the unique gene names comprising pDelVars of all animal species. Duplicated genes within species were previously removed.

Multiple genomic alignments of the selected genes were performed to find orthoVars, which we termed orthoVars, and potentially deleterious orthoVars, referred to as orthopDelVars. Genes comprising pDelVars in the highest number of species and genes with the highest number of pDelVars were further analyzed using Ensembl/Genomic alignments/ multiple alignments, option 18 eutherian mammals EPO (Enredo, Pecan, Ortheus). The option 18 eutherian mammals EPO includes eight species, examined in the present study (human, pig, cattle, mouse, rat, sheep, dog, and horse), how- ever, zebrafish is not included; therefore, genomic alignments for zebrafish were performed separately using pairwise alignments. Variants were considered as orthologous if they were located at the same nucleotide position or in the same nucleotide triplet (codon) within the orthologous gene of at least two species.

\section{In silico database screening for function}

We conducted literature and database screening for function, associated with pDelVars and associated genes in several databases: PubMed (https://www.ncbi.nlm.nih.gov/pubmed), Web of Science (https://login.webofknowledge.com), ScienceDirect (www.sciencedirect.com), and the database for annotation, visualization, and integrated discovery (DAVID) tool (https://david.ncifcrf.gov/tools.jsp).

In addition, we obtained information on clinical significance (ClinVar) of the variants using Ensembl/BioMart. Clinical significance terms applied in BioMart are the following: association, benign, confers sensitivity, drug response, likely benign, likely pathogenic, not provided, other, pathogenic, protective, risk factor, and uncertain significance. A list of human variants in each chromosome was uploaded in BioMart separately and then filtered for clinical significance. The frequency of variant occurrence was determined with Ensembl (www.ensembl.org/Homo_sapiens/ Info/Annotation).

Furthermore, pathway enrichment analysis for the human genes with pDelVars $(15,846)$ was conducted using the DAVID tool (Jiao et al., 2012). Background genes for the calculation of $p$-values were set as default and comprise corresponding genome-wide genes with at least one annotation in the analyzing categories. Similarly, for the genes with pDelVars and genes selected according to database screening biological pathways (Kyoto Encyclopedia of Genes and Genomes [KEGG] pathway, BIOCARTA) and diseases (Genetic Association Database [GAD] diseases, Online Mendelian Inheritance in Man [OMIM], diseases) in human were identified. Finally, we also extracted main annotated functions and biological processes using the Ensembl's Ontologies.

\section{Results}

Genome-wide prioritization of variants with predicted deleterious effect on protein function

Genome-wide prioritization of variants using the BioMart tool resulted, to the best of our knowledge, in the first catalog of pDelVars with predicted deleterious effect on protein function in nine vertebrate species. In zebrafish, mouse, rat, $\mathrm{dog}$, pig, sheep, horse, and cattle, genome-wide prioritization of pDelVars was performed using the SIFT tool. However, in human, pDelVars were prioritized for each individual chromosome separately using both, the SIFT and the PolyPhen tool, and the data were then merged. We also extracted the information on experimentally validated pDelVars to obtain supporting evidences associated to a variant. The main steps and results of the study are described in the Figure 1A (genome-wide screening) and $\mathrm{B}$ (in silico func- $4 \mathrm{~F} 1$ tional analysis).

The graphical summary (Fig. 2) emphasizes the main re- 4 F2 sults of the study. Human NEB (nebulin) gene encompasses 
A

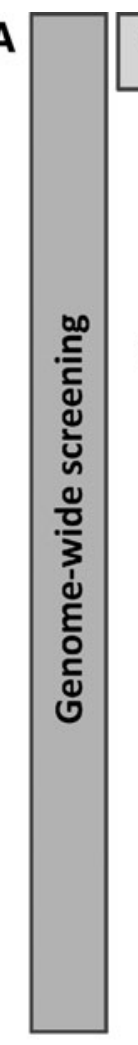

Tools
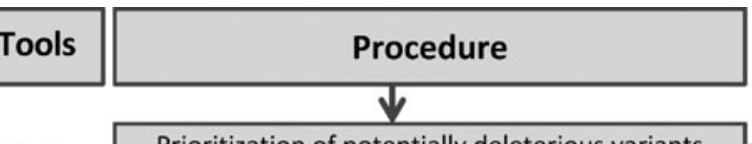

BioMart

Prioritization of potentially deleterious variants in 9 vertebrate species.

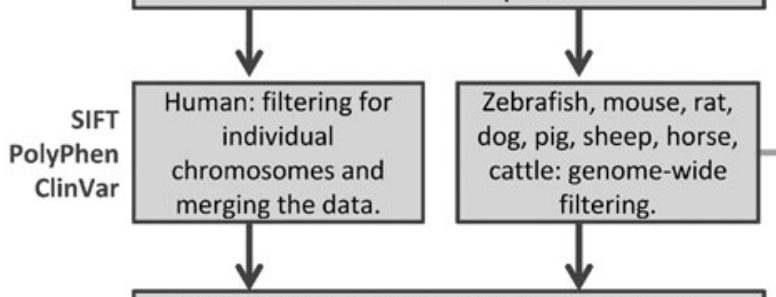

BioMart Obtaining gene names, gene descriptions and associated phenotypes.
B



\section{Tools}



Davis

DAVID analysis of genes with potentially deleterious variants in human (15846 genes)

\section{$\Rightarrow$
$\Rightarrow$}

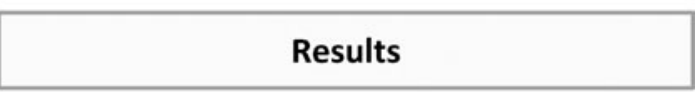

Catalog of 812273 missense potentially deleterious variants in 9 vertebrate species. Examples:

- mouse: 0.01\% (8 790/ 80104013$)$

- cattle: $0.57 \%$ (5 646 36/ 99159603 )

- human: 0.12\% (190 547/ 153774 718); 1.83\%

(3 483/190 547) with pathogenic clinical significance

Genes with the highest number of potentially deleterious variants in: human: $N E B(n=327)$, pig: TRRAP $(n=31)$, mouse: Obscn $(n=21)$, sheep: SHROOM2 $(n=20)$, cattle: LAMA3 $(n=18)$

Genes with potentially deleterious variants that occur in 7 of 9 examined species: - MPDZ

- TACC2

Orthologous potentially deleterious variants in a gene:

- MPDZ: human: rs150393677, dog: rs22153300 - MPDZ: human: rs779688439, mouse: rs28101990 - TACC2: human: rs756574052, cattle: rs455330779 - TACC2: human: rs761622230, cattle: rs479667924 - TLR7: human: rs34501186, cattle: rs133254244

FIG. 1. Overview of the study. The main objective of the present study was to prioritize variants with the most deleterious effect on protein function in nine animal genomes and extract their functional annotation from various databases. (A) Workflow and main results of the genome-wide screening for pDelVars. (B) Workflow and main results of the in silico functional analysis. DAVID, the database for annotation, visualization, and integrated discovery; pDelVars, potentially deleterious variants; SIFT, sorts intolerant from tolerant.

the highest number of pDelVars. Genes $M P D Z$ (multiple PDZ domain crumbs cell polarity complex component) and TACC2 (encoding transforming acidic coiled-coil containing protein 2) comprise pDelVars in seven out of nine examined species. The genes are, however, annotated in all nine species. In addition, five ortho-pDelVars were discovered in selected genes and are marked with vertical dotted lines.
The outcome of prioritization of pDelVars in nine vertebrate genomes is summarized in Table 1 . In total, the genomes of nine vertebrate species comprise 478,280,319 variants, of which $812,273(0.17 \%)$ confer potentially deleterious phenotype. The highest number of all known variants was found in human $(153,774,718)$, whereas the lowest was in rat $(4,915,429)$. Similarly, the variant frequency (on average 


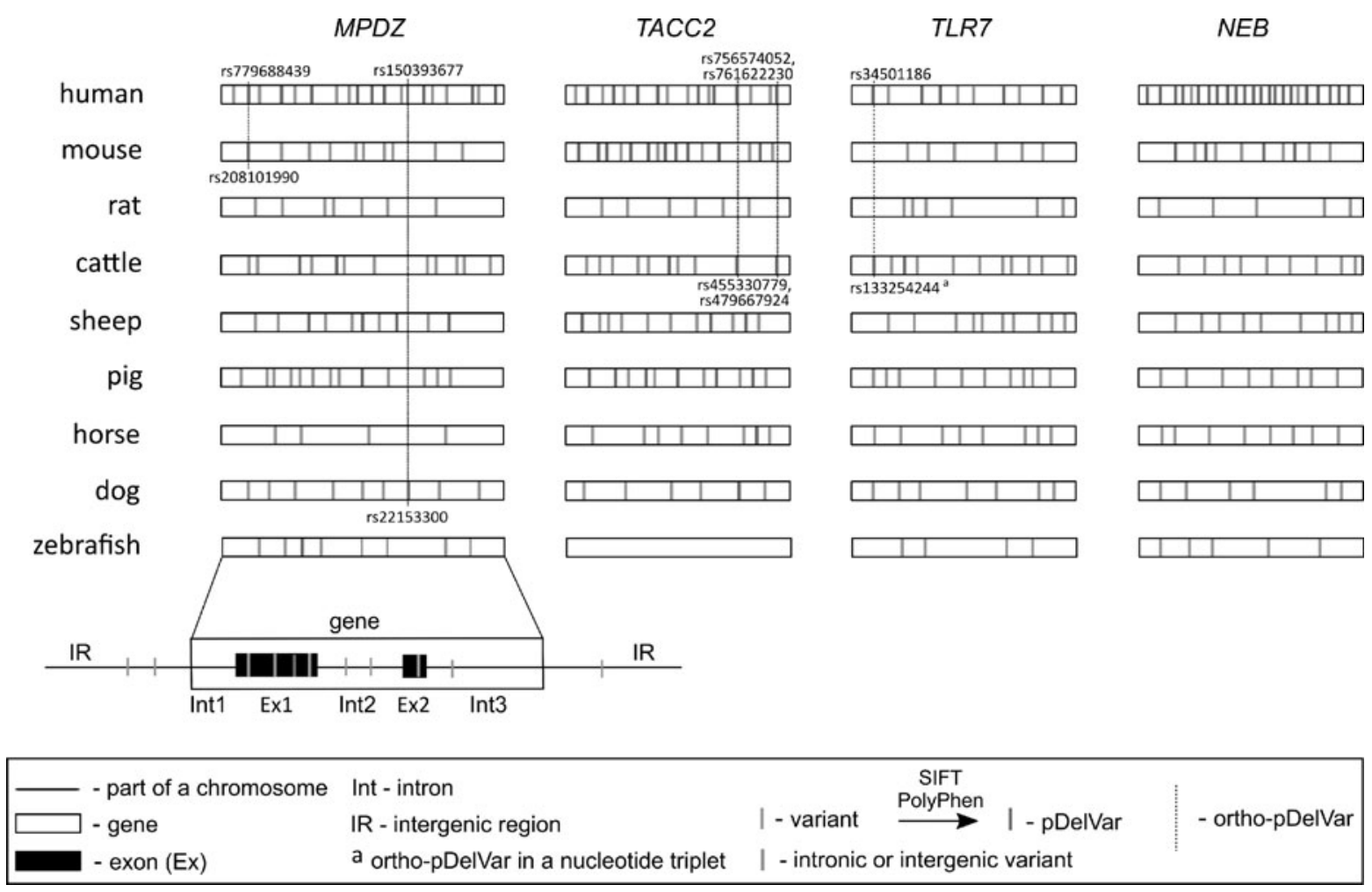

FIG. 2. Graphical summary of the study. The study presents the first catalog of missense variants with predicted deleterious effect on protein function (pDelVars) in nine vertebrate species. pDelVars with deleterious SIFT prediction (SIFT score $=0.0)$ were prioritized using BioMart, whereas in human, PolyPhen tool was applied as well (PolyPhen score $=1.0$; probably damaging). Multiple genomic alignments of the MPDZ, TACC2, TLR7, and NEB genes among nine vertebrates are presented. Genes are not drawn to scale; all orthologous genes are drawn with the same length and only coding regions are shown. Variants in exons that are predicted to be benign, tolerated, unknown, or possibly damaging are shown in green. On the contrary, variants with predicted deleterious effect on protein function (pDelVars) are presented in red. The number of red lines is proportional to the number of pDelVars, with exception of human MPDZ, TACC2, and NEB genes, which comprise a high number of variants (142, 42, and 327, respectively). Intronic and intergenic variants are shown in gray. Five orthologous ortho-pDelVars that are located at the same nucleotide position or in the same codon in genomes of two (or more) species were discovered in: (a) MPDZ gene: (1) dog (rs22153300) and human (rs150393677) and (2) human (rs779688439) and mouse (rs28101990), (b) TACC2 gene: (3) human (rs756574052) and cattle (rs455330779) and (4) human (rs761622230) and cattle (rs479667924), and (c) TLR7 gene: (5) cattle (rs34501186) and human (rs133254244). $M P D Z$ and TACC2 genes comprise pDelVars in seven out of nine examined species, and TLR7 an NEB gene in two. There are no known variants in zebrafish TACC2 gene. Furthermore, human $N E B$ gene comprises the highest number of pDelVars. ortho-pDelVars, potentially deleterious variants; PolyPhen, polymorphism phenotyping; SIFT, sorts intolerant from tolerant.

one variant per $23 \mathrm{bp} ; 3,547,762,741 \mathrm{bp} / 153,774,718$ variants) and the number of annotated genes with pDelVars $(15,869)$ are the highest in human genome and the lowest in rat, which carries in its genome one variant per $618 \mathrm{bp}$ $(3,042,335,753 \mathrm{bp} / 4,915,429$ variants) and only 48 annotated genes with pDelVars.

In each species, there are on average 90,253 pDelVars. The highest number $(564,636)$ as well as percentage $(0.57 \%$, $564,636 / 99,159,603)$ of pDelVars was identified in cattle, where 174,779 out of 564,636 are experimentally validated. Rat genome encompasses the lowest number of pDelVars (1517; of which only 51 are experimentally validated), whereas the lowest percentage of pDelVars was identified in mouse genome $(0.01 \%, 8790 / 80,104,013)$.

Regarding human, genome-wide data extraction by BioMart is no longer possible due to a large amount of genomic information. The pDelVars were therefore prioritized for each chromosome separately and the data then merged. The number of pDelVars according to each individual human chromosome can be found in Supplementary Table S1.

Altogether, $0.12 \%(190,547 / 153,774,718)$ of human variants is predicted to have deleterious effect on protein function. Of these, $91.72 \%$ pDelVars $(174,779 / 190,547)$ are experimentally validated and $1.83 \%(3483 / 190,547)$ carries pathogenic clinical significance. The absolute number of pDelVars is the highest in chromosome $1(18,824)$ and the lowest in chromosome Y (55) (Supplementary Fig. S1). In the $\varangle$ SF1 latter, the ratio between the number of pDelVars and total number of sequence variants is the lowest (0.000263).

On the contrary, human chromosome 19 comprises the highest number of pDelVars relative to the total number of sequence variants (0.0033) (Supplementary Fig. S1). The number of pDelVars per bp of the chromosome is consequently the highest in chromosome $19(0.0002)$ and the lowest in chromosome Y $\left(9.6 \times 10^{-07}\right)$ (Supplementary Fig. S1). Moreover, we investigated the ratio between the number of 


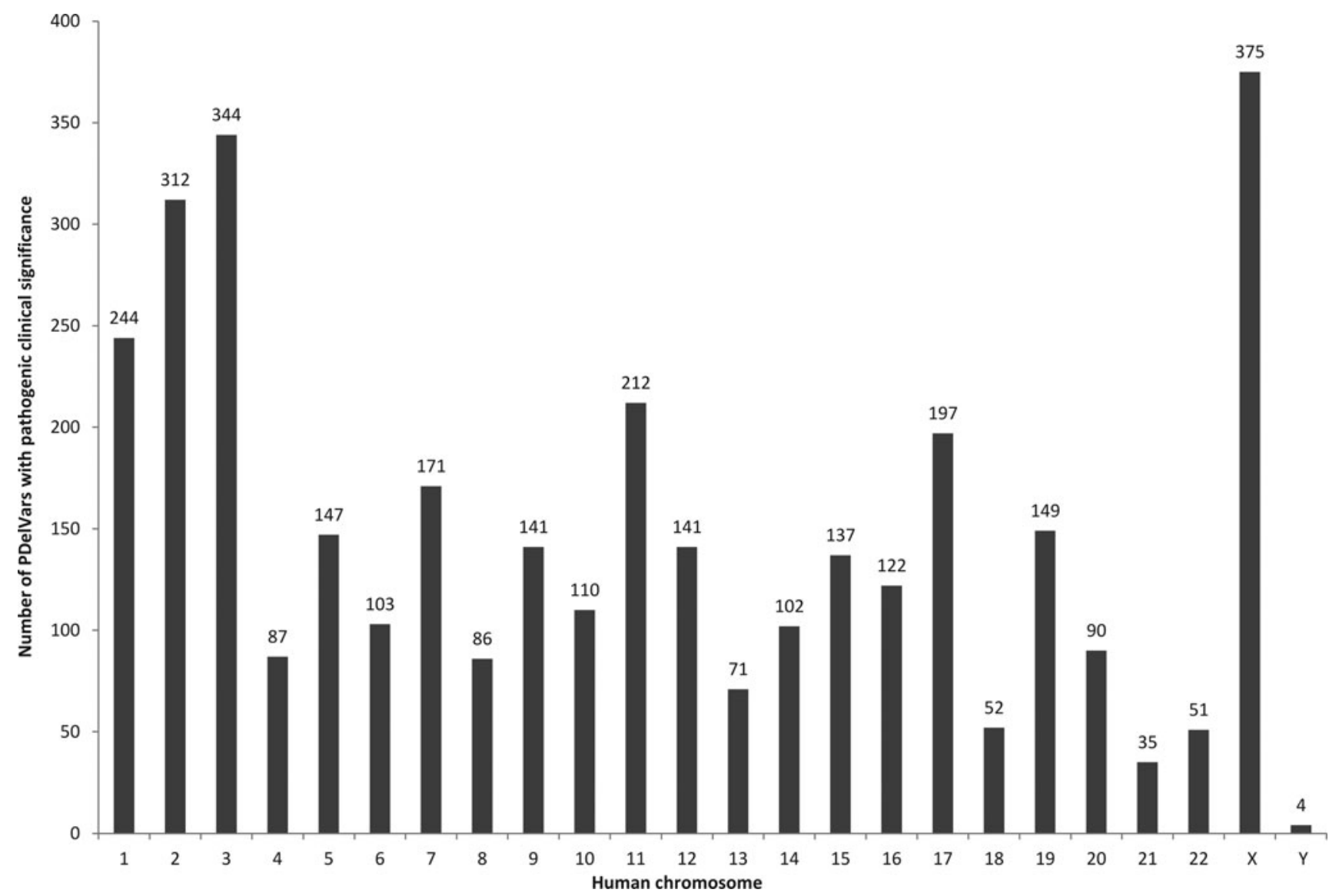

FIG. 3. Number of pDelVars with pathogenic clinical significance for each human chromosome.

pDelVars with pathogenic clinical significance and the total number of pDelVars and the results revealed that chromosome 4 presents the lowest ratio $(103 / 9820,1.05 \%)$ and chromosome $X$ the highest $(375 / 3901 ; 9.6 \%)$. The number of pDelVars with pathogenic clinical significance according to

F3 each human chromosome is provided in Figure 3.

The catalogs of human and animal pDelVars, which are ST2 experimentally validated, are available in Supplementary ST3 Tables $\mathrm{S} 2$ and $\mathrm{S} 3$, respectively.

\section{Genes with the highest number of pDelVars}

Genes comprising the highest number of pDelVars within each individual species and genes that comprise pDelVars in the highest number of species (seven out of nine examined) were determined using a custom Python script. Genes with the highest number of pDelVars within each individual species

$\mathrm{SF} 2$ are presented in Supplementary Figure S2. The human NEB gene comprises the highest number of pDelVars $(n=327)$, followed by OBSCN (199), DNAH3 (189), DNAH8 (180), and USH2A (179) genes. The number of pDelVars is significantly lower in genes of the other species. TRRAP gene in pig, encoding for the transformation/transcription domain associated protein, encompasses 31 pDelVars. Genomes of dog, rat, and horse, however, contain the lowest number $(n=2)$ of pDelVars in a gene.

Results revealed that MPDZ (multiple PDZ domain crumbs cell polarity complex component) and TACC2 (transforming acidic coiled-coil containing protein 2) genes comprise pDelVars in seven out of nine examined species (Table 2). Both genes are annotated in genomes of all nine $4 \mathrm{~T} 2$ species; however, pDelVars are present in seven out of nine examined species. pDelVars in MPDZ lack in genomes of horse and rat, and similarly, pDelVars in TACC2 are absent in genomes of rat and zebrafish. Moreover, we also identified genes with pDelVars that occur in six out of nine species: AKAP11, ALPK2, C2CD3, CD101, CDHR2, CUL9, DCHS2, DIS3 L, FAM208B, FAN1, FBF1, JMJD1C, LMTK2, MYO5C, PLA2R1, QSOX1, RUSC2, SFI1, TG, TLR5, VCAN, and XKR5 (Table 2).

\section{Identification of the ortho-pDe/Vars}

To identify interspecies ortho-pDelVars and orthoVars, multiple genomic alignments were constructed for six selected genes with pDelVars, namely two genes that comprise pDelVars in the highest number of species (MPDZ, TACC2), two genes with the highest number of pDelVars $(N E B$, $T R R A P)$, a previously reported gene with orthoVars $(R Y R I)$, and a previously reported gene with pDelVars (TLR7). Five ortho-pDelVars were detected in the following three genes: $M P D Z, T A C C 2$, and TLR7.

In detail, $M P D Z$ gene comprises two ortho-pDelVars: (1) $\operatorname{dog}(\mathrm{rs} 22153300)$ and human (rs150393677) and (2) mouse (rs28101990) and human (rs779688439) (Table 3, Supple- 4 T3 4 SF3 mentary Fig. S3). The four nsSNPs are predicted to have deleterious effect on protein function (SIFT score $=0.0$ and $/$ or PolyPhen score $=1.0$ ). 
Table 2. Genes Comprising Potentially Deleterious Variants in the Highest Number of Examined Species

\begin{tabular}{|c|c|c|c|c|c|}
\hline \multirow[b]{2}{*}{ Species (scientific name) } & \multicolumn{5}{|c|}{ Genes } \\
\hline & $M P D Z$ & $T A C C 2$ & $\begin{array}{c}\text { AKAP1, ALPK2, C2CD3, } \\
\text { CD101, CDHR2, CUL9, } \\
\text { FBF1, TLR5, MYO5C, } \\
\text { RUSC2, SFI1, VCAN, XKR5 }\end{array}$ & $\begin{array}{c}\text { DCHS2, FAN1, } \\
\text { LMTK2, } \\
\text { PLA2R1, TG }\end{array}$ & $\begin{array}{l}\text { DIS3L, FAM208B, } \\
\text { JMJD1C, QSOX1 }\end{array}$ \\
\hline $\begin{array}{l}\text { D. rerio } \\
\text { M. musculus }\end{array}$ & & & & & \\
\hline $\begin{array}{l}R . \text { norvegicus } \\
C . \text { familiaris }\end{array}$ & & & & & \\
\hline S. scrofa & & & & & \\
\hline $\begin{array}{l}O \text {. aries } \\
\text { E. caballus }\end{array}$ & & & & & \\
\hline $\begin{array}{l}\text { E. caballus } \\
\text { B. taurus }\end{array}$ & & & & & \\
\hline $\begin{array}{l}\text { B. taurus } \\
\text { H. sapiens }\end{array}$ & & & & & \\
\hline
\end{tabular}

Dark gray color pDelVars present

Light gray color pDelVars absent

Furthermore, we also detected several orthoVars in $M P D Z$ gene (Table 3). An orthoVar consists of a pDelVar (SIFT score $=0.0$ and $/$ or PolyPhen score $=1.0$ ) and a missense nsSNP that has a SIFT score greater than 0.0 and/or a PolyPhen score lower than 1.0. In TACC2 gene, we identified several orthoVars and two ortho-pDelVars (Table 3). The latter comprise the following nsSNPs: (1) human (rs756574052) and cattle (rs455330779), (2) human (rs761622230) and cattle (rs479667924) (Supplementary Fig. S3).

Finally, we identified ortho-pDelVars and orthoVars in two selected genes with pDelVars (RYR1, TLR7), chosen according to database screening. We detected an orthopDelVar in TLR7 gene, encoding toll-like receptor 7, of cattle (rs34501186) and human (rs133254244), where the nsSNPs are located in the same codon (Table 3, Supplementary Fig. S3). Human $R Y R 1$ gene, encoding the ryanodine receptor 1 , comprises 104 pDelVars. We found that a pDelVar in human (rs193922839) RYRl gene has an orthologous variant in cattle (rs474030293) (Table 3, Supplementary Fig. S3).

\section{Analysis of associated biological} pathways and diseases

Pathway enrichment analysis for human genes with T4 pDelVars $(15,846)$ was performed (Table 4). Results showed that genes containing pDelVars are most significantly associated with the two biological pathways: metabolic pathways $\left(1108 / 15,846\right.$ genes with pDelVars; $p$-value: $\left.1.1 \times 10^{-20}\right)$ and neuroactive ligand-receptor interaction $(260 / 15,846$ genes with pDelVars; $p$-value: $3.3 \times 10^{-9}$ ); and the two diseases: tobacco use disorder $(2729 / 15,846$ genes; $p$-value: $\left.2.9 \times 10^{-54}\right)$ and type 2 diabetes $(1952 / 15,846$ genes; $p$-value: $\left.1.2 \times 10^{-28}\right)$.

For the genes that comprise pDelVars in the highest number of species (MPDZ, TACC2), a gene with the highest number of pDelVars $(N E B)$, and genes selected according to the literature (RYR1, TLR7) main annotated functions, biological processes, biological pathways, and diseases were extracted. Results showed that TLR7 gene has an important role in immune system, since it is associated with the toll-like receptor pathway. Results also suggest that it can contribute to numerous diseases, many of them autoimmune (e. g. rheumatoid arthritis, asthma, atherosclerosis, lupus erythematosus, and multiple sclerosis). MPDZ gene, which plays a role in tight junction, contributes to alcohol consumption and alcohol withdrawal seizures. TACC2 gene is associated with nuclear hormone receptor binding, tobacco use disorder, Alzheimer's disease, and breast cancer. RYRl gene is an important component of calcium signaling pathway that plays a role in myopathies, tobacco use disorder, and malignant hyperthermia $(M H)$ syndrome. Finally, $N E B$ gene is associated with actin binding and regulation, body height, and type 2 diabetes.

\section{Discussion}

In the present study, we performed in silico genome-wide screening for $\mathrm{pDelV}$ ars in nine vertebrate species. To the best of our knowledge, this study presents the first catalog of variants with the most deleterious effect on protein function in nine animal genomes. Moreover, we extracted functional annotation of the collected variants from various databases and identified several interspecies ortho-pDelVars.

The key findings of the study are emphasized in the graphical summary (Figure 2), where the genes with pDelVars (TACC2, MPDZ, TLR7, NEB) are aligned among species. Furthermore, five ortho-pDelVars were discovered in selected genes and are marked with vertical dotted lines.

Human genome contains an average of one variant every 23 bases, of which $0.12 \%$ are predicted to be deleterious. Lek et al. (2016) reported one variant every eight bases of the human exome. The highest frequency of pDelVars was, however, found in cattle $(0.57 \%)$ and the lowest in mouse $(0.01 \%)$. The highest number of annotated genes with pDelVars was identified in human $(15,846)$, whereas the lowest in rat (48). High discrepancy in the number of annotated genes and variants between species is most likely not due to biological reason, but differences in progress of sequencing and annotation projects. Pathogenic clinical significance data of pDelVars in human that were extracted using BioMart showed that $1.83 \%$ of pDelVars carries pathogenic clinical significance. 


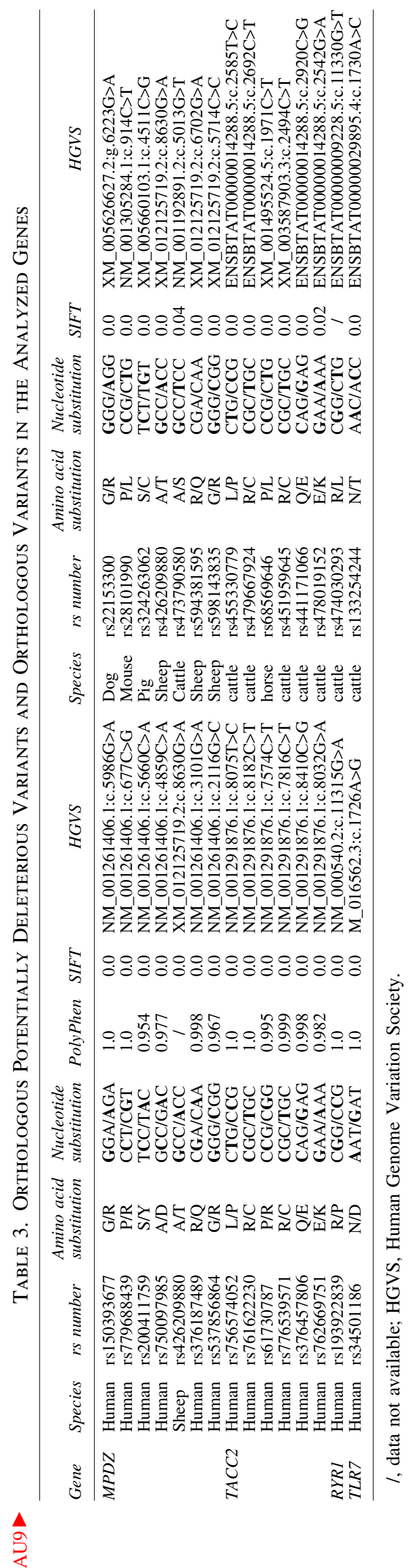

It is also important to note that sequencing data were obtained in several projects with possible different sequence coverage that affects variant identification. Low-coverage sequencing generates genotypes with higher error rate resulting in a higher number of false-positive variants. Abe- 4 AU4 casis et al. (2011), however, reported that low-coverage sequencing $(2-6 \times)$ efficiently identified shared variants on common haplotypes in human, but had lower power to detect rare haplotypes and associated variants coupled with more inaccurate genotypes (error rate: 1-3\%) (1000 Genomes Project Consortium et al., 2015).

Furthermore, genes with the highest number of pDelVars within each individual species and genes that comprise pDelVars in the highest number of species were determined. $M P D Z$ and TACC2 genes comprise pDelVars in seven out of nine examined species. There may indeed be a biological explanation for these two genes to be more vulnerable; however, data regarding the genetic variants are not yet complete in the database and the result could change in the future.

Human $N E B$ gene comprises the highest number of pDelVars $(n=327)$. Studies show that $N E B$ gene plays an important role in numerous cellular processes, including regulation of muscle contraction, Z-disc formation, and myofibril organization and assembly (Chu et al., 2016). Variations in this gene were reported to be associated with different myopathies (Gajda et al., 2015; Malfatti et al., 2015). Opposed to human, genomes of the other species encompass fewer pDelVars in a single gene, which is most probably due to the fact that they are not yet annotated as well as the human genome. In fact, some genes do not yet have Ensembl IDs.

\section{Orthologous pDelVars}

Altogether, we identified five ortho-pDelVars in the three genes, namely MPDZ, TACC2, and TLR7. Two were discovered in MPDZ gene: (1) dog (rs22153300) and human (rs150393677) and (2) mouse (rs28101990) and human (rs779688439). Their function is not yet annotated and should be investigated in the future. Saugier-Veber et al. (2017) reported that MPDZ gene is a component of tight junctions, which are expressed from early brain development in choroid plexuses and ependyma. Studies implicate that $M P D Z$ gene sequence and/or expression have an important role in ethanol withdrawal in mice as well as alcoholism in humans (Kruse et al., 2014; Milner et al., 2015), which was confirmed with DAVID tool as well. MPDZ gene variants are known contributors of acute alcohol withdrawal severity and seizures in mice (Fehr et al., 2002), whereas sequencing of MPDZ gene in individuals with European American ancestry revealed no variants in the sites identical to those associated with alcohol withdrawal seizures in mice. Still, variants in human $M P D Z$ gene suggest a possible association with alcohol dependence (Karpyak et al., 2009). Variants in MPDZ have also been associated with congenital hydrocephalus, a neuropathy (Feldner et al., 2017; Saugier-Veber et al., 2017).

Another two ortho-pDelVars were found in TACC2 gene: (1) human (rs756574052) and cattle (rs455330779) and (2) human (rs761622230) and cattle (rs479667924). TACC2 gene encodes transforming acidic coiled-coil-containing protein 2, which is associated with the centrosome-spindle apparatus during cell cycling and division, and is expressed in 


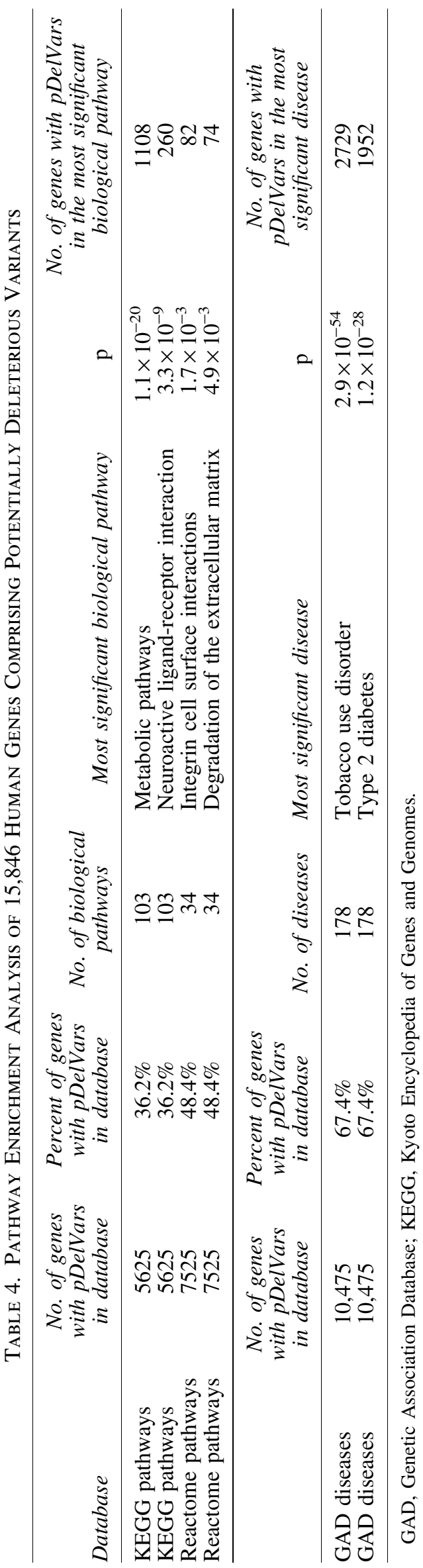

postmitotic tissues, including heart, muscle, kidney, and brain (Cheng et al., 2010). Due to its important role in cell division, it is not surprising that it might play a role in some forms of cancer (Ha et al., 2013; Lauffart et al., 2003), particularly in prostate (Takayama et al., 2012) and breast cancer (Onodera et al., 2016). According to DAVID tool analysis, TACC2 gene plays a role in Alzheimer's disease, breast cancer, and tobacco use disorder. Interestingly, the pathway enrichment analysis of genes with pDelVars in human showed that the most significant disease, in which the most genes with pDelVars $(2729 / 15,846)$ participate, is tobacco use disorder. The second most significant disease is type 2 diabetes (1952/15,846 genes with pDelVars). We also reported the most significant biological pathways in which genes with pDelVars participate: metabolic pathways (1108/ 15,846 genes with pDelVars) and neuroactive ligand-receptor interaction (260/15,846 genes with pDelVars).

The remaining ortho-pDelVar was discovered in the codon of TLR7 gene in cattle (rs34501186) and human (rs133254244). The human variant was previously associated with significant loss of function in TLR7 (Colak et al., 2014) that has an important role in immune system (Barchet et al., 2005; Gantier et al., 2008). TLR7 gene is according to DAVID analysis associated with a number of various diseases, for example arthritis, atherosclerosis, celiac disease, lupus erythematosus, multiple sclerosis, and virus diseases.

$R Y R 1$ gene presents an important model for the study of orthoVars. There is evidence that an orthologous variant in the same codon of RYRl gene in human and pig causes the same amino acid substitution and affects the MH phenotype (Fagerlund et al., 1994; Gillard et al., 1991; Hogan et al., 1992; Otsu et al., 1992). Given that several genes and numerous variants are involved in $\mathrm{MH}$ phenotype in human, the genetics of this disease is much more complex than in pig (Brandom and Callahan, 2015; Correia et al., 2012). However, an additional orthoVar was revealed in the RYRl gene in cattle (rs474030293) and human (rs193922839). The latter is thought to be associated with congenital myopathies (Monnier et al., 2008; Zhou et al., 2007). RYR1 is reported to be the largest ion channel protein known, which has a role in effective skeletal muscle contractile activation (HernándezOchoa et al., 2016).

Study of orthoVars is of importance and deserves a great attention in the future, since genetic causes for diseases by interspecies comparison can be identified. In fact, some studies of orthologous SNPs between human and chimpanzee were already conducted. Hodgkinson et al. (2009) reported that there is an excess of sites at which humans and chimpanzees have SNPs. Later, Hodgkinson and Eyre-Walker (2010) concluded that the heterogeneity in triplet frequencies is not specifically associated with coSNP, but is instead driven by base composition bias around $\mathrm{CpG}$ dinucleotides. An extensive genome-wide study, including five primate species, was carried out by Chen et al. (2016). They reported an excess of coSNPs at human-chimpanzee orthologous positions due to cryptic variation in the mutation rate. In our study, we extended the number of species to nine vertebrates, including nonprimate species. Since an amount of genomics data coupled with identified sequence variants is steadily increasing, more orthoVars are expected to be identified in the future. Analysis of orthoVars presents an opportunity to prioritize variants with probable effect on the phenotype. 


\section{Prioritization protocols for the genetic variants}

Protocols for prioritization of genetic variants with predicted functional effect differ between studies. For example, our previous protocols for variant prioritization were based on the genome structure. For example, we identified variants located within regions of overlaps between different genomic elements (coding and noncoding transcriptional units, quantitative locus traits [QTLs]) (Zorc and Kunej, 2016). In addition, we screened for variants, located within regions corresponding to predicted and validated protein interactions sites (Skrlj and Kunej, 2016; Skrlj et al., 2017). However, in the present study, the criteria for prioritization were predicted potentially deleterious effect on protein function.

In the future, protocols for prioritization should be improved with more criteria and elements included. Several novel tools for variant prioritization are being reported, for example M-cap (Jagadeesh et al., 2016) and PROVEAN (Choi and Chan, 2015) that will enable extended repertoire of analysis options and will be accessible in the future to include in the analysis. Given that most of the tools for variant prioritization are available only for human (e.g., the PolyPhen tool), and more tools for other species are required.

With rapidly increasing information on sequence variants, it is important to extract the desired information to annotate it and associate it with other types of genomic information. The variant gains true value only when it is associated with a phenotype, a QTL, a protein target, or a disease. Only then it becomes valuable and can be further implemented in various different applications, including drug discovery and diagnostic tests.

We developed a catalog of prioritized variants with predicted deleterious effect on protein function that might affect the phenotype and disease development and thus deserve further functional studies. Altogether, we extracted 812,273 missense pDelVars in nine vertebrate species. The catalog presents an important source of information for further studies and identification of orthoVars. The implementation of the protocol for prioritization using the BioMart tool is, however, immensely time consuming due to excessive amount of data, which will in the future only rapidly increase. Moreover, in human, genome-wide analysis is not possible. The pDelVars must therefore be prioritized for each chromosome separately, resulting in a tedious and unnecessarily time-consuming analysis. The catalog, thus, presents an extremely valuable source of information, collected in a handy format (Excel) that allows fast and simple acquisition of the desired information.

It is important to note that Ensembl browser is updated around four times per year due to increasing amount of new information. The SIFT and PolyPhen data are therefore changing and complementing between Ensemble database releases. The present study is ongoing and will have to be frequently updated according to novel releases of genomic browsers. Update studies will also reveal a higher number of orthoVars and associated genes. With a systematic study of all disease associated variants in animals and interspecies comparison using comparative genomics and orthoVars novel disease genes could be discovered. Our catalog of missense pDelVars is of great importance since it can be applied in numerous fields, for example, to research of candidate disease markers associated with polygenic diseases, such as
Parkinson's disease (Redenšek et al., 2018), personalized medicine, iatromics (Hekim and Özdemir, 2017), pharmacogenomics, nutrigenomics, ecogenomics (Ozdemir et al., 2009), and other omics types (Pirih and Kunej, 2017). Furthermore, the catalog may be applied in identification of pDelVars, located within miRNA-target interaction sites (Piletič and Kunej, 2017) or other types of molecular interactions. Collected genetic variants could also enable development of new commercial SNP chips and diagnostic tests.

\section{Executive Highlights}

We report here:

- systematic genome-wide prioritization of missense pDelVars in nine vertebrate species,

- the first catalog of missense variants with predicted deleterious effect in nine animal genomes,

- note that the highest frequency of pDelVars was detected in cattle $(0.57 \%)$, whereas the lowest in mouse $(0.01 \%)$. In humans, $0.12 \%$ of variants are predicted to have deleterious effect on protein function.

- $M P D Z$ and TACC2 genes comprise pDelVArs in the highest number of species (seven out of nine examined species).

- For certain selected genes, we also conducted interspecieswide identification of orthologous pDelVars, which might have an effect on phenotype and disease development and thus deserve further functional studies. Five ortho-pDelVars were discovered in three different genes.

- pDelVars and ortho-pDelVars, presented in our catalog, can be further used in numerous omics applications so as to harness genomics big data such as personalized medicine, epigenomics, nutrigenomics, pharmacogenomics, research on polygenic diseases, identification of candidate genes associated with common complex diseases, development of new clinically relevant SNP chips, drug discovery tools, and diagnostic companion tests to individualize drug treatment and other health interventions (iatromics).

\section{Acknowledgments}

This work was supported by the Slovenian Research Agency (ARRS) through the Research program Comparative genomics and genome biodiversity (grant number P4-0220).

\section{Author Disclosure Statement}

No competing financial interests exist.

\section{References}

1000 Genomes Project Consortium, Auton A, Brooks LD, et al. (2015). A global reference for human genetic variation. Nature $526,68-74$.

Adzhubei I, Jordan DM, and Sunyaev SR. (2013). Predicting functional effect of human missense mutations using PolyPhen-2. Curr Protoc Hum Genet 7, Unit7.20.

Adzhubei IA, Schmidt S, Peshkin L, et al. (2010). A method and server for predicting damaging missense mutations. Nat Methods 7, 248-249.

Balbi KJ, and Feil EJ. (2007). The rise and fall of deleterious mutation. Res Microbiol 158, 779-786. 
Barchet W, Krug A, Cella M, et al. (2005). Dendritic cells respond to influenza virus through TLR7- and PKR-independent pathways. Eur J Immunol 35, 236-242.

Brandom BW, and Callahan PM. (2015). Malignant hyperthermia: An update. Adv Anesth 33, 113-128.

Care MA, Bradford JR, Needham CJ, Bulpitt AJ, and Westhead DR. (2009). Combining the interactome and deleterious SNP predictions to improve disease gene identification. Hum Mutat 30, 485-492.

Chen CY, Hung LY, Wu CS, and Chuang TJ. (2016). Purifying selection shapes the coincident SNP distribution of primate coding sequences. Sci Rep 6, 27272.

Cheng S, Douglas-Jones A, Yang X, Mansel RE, and Jiang WG. (2010). Transforming acidic coiled-coil-containing protein 2 (TACC2) in human breast cancer, expression pattern and clinical/ prognostic relevance. Cancer Genomics Proteomics 7, 67-73.

Choi Y, and Chan AP. (2015). PROVEAN web server: A tool to predict the functional effect of amino acid substitutions and indels. Bioinformatics 31, 2745-2747.

Chu M, Gregorio CC, and Pappas CT. (2016). Nebulin, a multifunctional giant. J Exp Biol 219, 146-152.

Colak E, Leslie A, Zausmer K, et al. (2014). RNA and imidazoquinolines are sensed by distinct TLR7/8 ectodomain sites resulting in functionally disparate signaling events. J Immunol 192, 5963-5973.

Correia ACC, Silva PCB, and da Silva BA. (2012). Malignant hyperthermia: Clinical and molecular aspects. Rev Bras Anestesiol 62, 820-837.

Cunningham F, Amode MR, Barrell D, et al. (2015). Ensembl 2015. Nucleic Acids Res 43, 662-669.

Fagerlund T, Ording H, Bendixen D, and Berg K. (1994). Search for three known mutations in the RYRI gene in 48 Danish families with malignant hyperthermia. Clin Genet 46, 401-404.

Fehr C, Shirley RL, Belknap JK, Crabbe JC, and Buck KJ. (2002). Congenic mapping of alcohol and pentobarbital withdrawal liability loci to a $<1$ centimorgan interval of murine chromosome 4: Identification of Mpdz as a candidate gene. J Neurosci 22, 3730-3738.

Feldner A, Adam MG, Tetzlaff F, et al. (2017). Loss of Mpdz impairs ependymal cell integrity leading to perinatal-onset hydrocephalus in mice. EMBO Mol Med 9, 890-905.

Gajda A, Horváth E, Hortobágyi T, et al. (2015). Nemaline myopathy type 2 (NEM2): Two novel mutations in the nebulin (NEB) gene. J Child Neurol 30, 627-630.

Gantier MP, Tong S, Behlke MA, et al. (2008). TLR7 is involved in sequence-specific sensing of single-stranded RNAs in human macrophages. J Immunol 180, 2117-2124.

Gillard EF, Otsu K, Fujii J, et al. (1991). A substitution of cysteine for arginine 614 in the ryanodine receptor is potentially causative of human malignant hyperthermia. Genomics 11, 751-755.

Ha GH, Kim JL, and Breuer EKY. (2013). Transforming acidic coiled-coil proteins (TACCs) in human cancer. Cancer Lett $336,24-33$.

Hekim N, and Özdemir V. (2017). A general theory for 'post' systems biology: Iatromics and the environtome. OMICS 21, 359-360.

Hernández-Ochoa EO, Pratt SJP, Lovering RM, and Schneider MF. (2016). Critical role of intracellular RyR1 calcium release channels in skeletal muscle function and disease. Front Physiol 6, 420.

Hodgkinson A, and Eyre-Walker A. (2010). The genomic distribution and local context of coincident SNPs in human and chimpanzee. Genome Biol Evol 2, 547-557.
Hodgkinson A, Ladoukakis E, and Eyre-Walker A. (2009). Cryptic variation in the human mutation rate. PLoS Biol 7, e1000027.

Hogan K, Couch F, Powers PA, and Gregg RG. (1992). A cysteine-for-arginine substitution (R614C) in the human skeletal muscle calcium release channel cosegregates with malignant hyperthermia. Anesth Analg 75, 441-448.

Jagadeesh KA, Wenger AM, Berger MJ, et al. (2016). M-CAP eliminates a majority of variants of uncertain significance in clinical exomes at high sensitivity. Nat Genet 48, 1581-1586.

Jiao X, Sherman BT, Huang da W, et al. (2012). DAVID-WS: A ४ AU5 stateful web service to facilitate gene/protein list analysis. Bioinformatics 28, 1805-1806.

Karpyak VM, Kim JH, Biernacka JM, et al. (2009). Sequence variations of the human MPDZ gene and association with alcoholism in subjects with European Ancestry. Alcohol Clin Exp Res 33, 712-721.

Kruse LC, Walter NAR, and Buck KJ. (2014). MPDZ expression in the caudolateral substantia nigra pars reticulata is crucially involved in alcohol withdrawal. Genes Brain Behav 13, 769-776.

Kumar P, Henikoff S, and Ng PC. (2009). Predicting the effects of coding non-synonymous variants on protein function using the SIFT algorithm. Nat Protoc 4, 1073-1082.

Lauffart B, Gangisetty O, and Still IH. (2003). Molecular cloning, genomic structure and interactions of the putative breast tumor suppressor TACC2. Genomics 81, 192-201.

Lek M, Karczewski KJ, Minikel EV, et al. (2016). Analysis of protein-coding genetic variation in 60,706 humans. Nature 536, 285-291.

Makino T, Rubin CJ, Carneiro M, Axelsson E, Andersson L, and Webster MT. (2018). Elevated proportions of deleterious genetic variation in domestic animals and plants. Genome Biol Evol 10, 276-290.

Malfatti E, Monges S, Lehtokari VL, et al. (2015). Bilateral footdrop as predominant symptom in nebulin (NEB) gene related 'core-Rod' congenital myopathy. Eur J Med Genet 58, 556-561.

Milner LC, Shirley RL, Kozell LB, et al. (2015). Novel MPDZ/ MUPP1 transgenic and knockdown models confirm Mpdz's role in ethanol withdrawal and support its role in voluntary ethanol consumption. Addict Biol 20, 143-147.

Monnier N, Marty I, Faure J, et al. (2008). Null Mutations causing depletion of the type 1 ryanodine receptor (RYR1) are commonly associated with recessive structural congenital myopathies with cores. Hum Mutat 29, 670-678.

Ng PC, and Henikoff S. (2001). Predicting deleterious amino acid substitutions predicting deleterious amino acid substitutions. Genome Res 11, 863-874.

Onodera Y, Takagi K, Miki Y, et al. (2016). TACC2 (transforming acidic coiled-coil protein 2) in breast carcinoma as a potent prognostic predictor associated with cell proliferation. Cancer Med 5, 1973-1982.

Otsu K, Phillips SM, Khanna KV, de Leon S, and MacLennan HD. (1992). Refinement of diagnostic assays for a probable causal mutation for porcine and human malignant hyperthermia. Genomics 13, 835-837.

Ozdemir V, Motulsky AG, Kolker E, and Godard B. (2009). Genome-environment interactions and prospective technology assessment: Evolution from pharmacogenomics to nutrigenomics and ecogenomics. OMICS 13, 1-6.

Piletič K, and Kunej T. (2017). Minimal standards for reporting microRNA:target interactions. OMICS 21, 197-206.

Pirih N, and Kunej T. (2017). Toward a taxonomy for multi-omics science? Terminology development for whole genome study approaches by omics technology and hierarchy. OMICS 21, 1-16. 
Redenšek S, Dolžan V, and Kunej T. (2018). From genomics to omics landscapes of Parkinson's disease: Revealing the molecular mechanisms. OMICS 21, 1-16.

Sachidanandam R, Weissman D, Schmidt SC, et al. (2001). A map of human genome sequence variation containing 1.42 million single nucleotide polymorphisms. Nature 409, 928-933.

Saugier-Veber P, Marguet F, Lecoquierre F, et al. (2017). Hydrocephalus due to multiple ependymal malformations is caused by mutations in the MPDZ gene. Acta Neuropathol Commun 5, 36.

Skrlj B, Konc J, and Kunej T. (2017). Identification of sequence variants within experimentally validated protein interaction sites provides new insights into molecular mechanisms of disease development. Mol Inform 36.

Skrlj B, and Kunej T. (2016). Computational identification of non-synonymous polymorphisms within regions corresponding to protein interaction sites. Comput Biol Med 79, 30-35.

Takayama K, Horie-Inoue K, Suzuki T, et al. (2012). TACC2 is an androgen-responsive cell cycle regulator promoting androgen-mediated and castration-resistant growth of prostate cancer. Mol Endocrinol 26, 748-761.

Wong KC, and Zhang Z. (2014). SNPdryad: Predicting deleterious non-synonymous human SNPs using only orthologous protein sequences. Bioinformatics 30, 1112-1119.

Xue Y, Chen Y, Ayub Q, et al. (2012). Deleterious- and disease-allele prevalence in healthy individuals: Insights from current predictions, mutation databases, and population-scale resequencing. Am J Hum Genet 91, 1022-1032.

Zhou H, Jungbluth H, Sewry CA, et al. (2007). Molecular mechanisms and phenotypic variation in RYR1-related congenital myopathies. Brain 130, 2024-2036.

Zorc M, and Kunej T. (2016). In silico screening of the chicken genome for overlaps between genomic regions: microRNA genes, coding and non-coding transcriptional units, QTL, and genetic variations. Chromosome Res 24, 225-230.
Address correspondence to:

Tanja Kunej, PhD

Department of Animal Science

Biotechnical Faculty

University of Ljubljana

Domžale 1230

Slovenia

E-mail: tanja.kunej@bf.uni-lj.si

\begin{tabular}{|c|}
\hline  \\
\hline
\end{tabular}




\section{Supplementary Data}

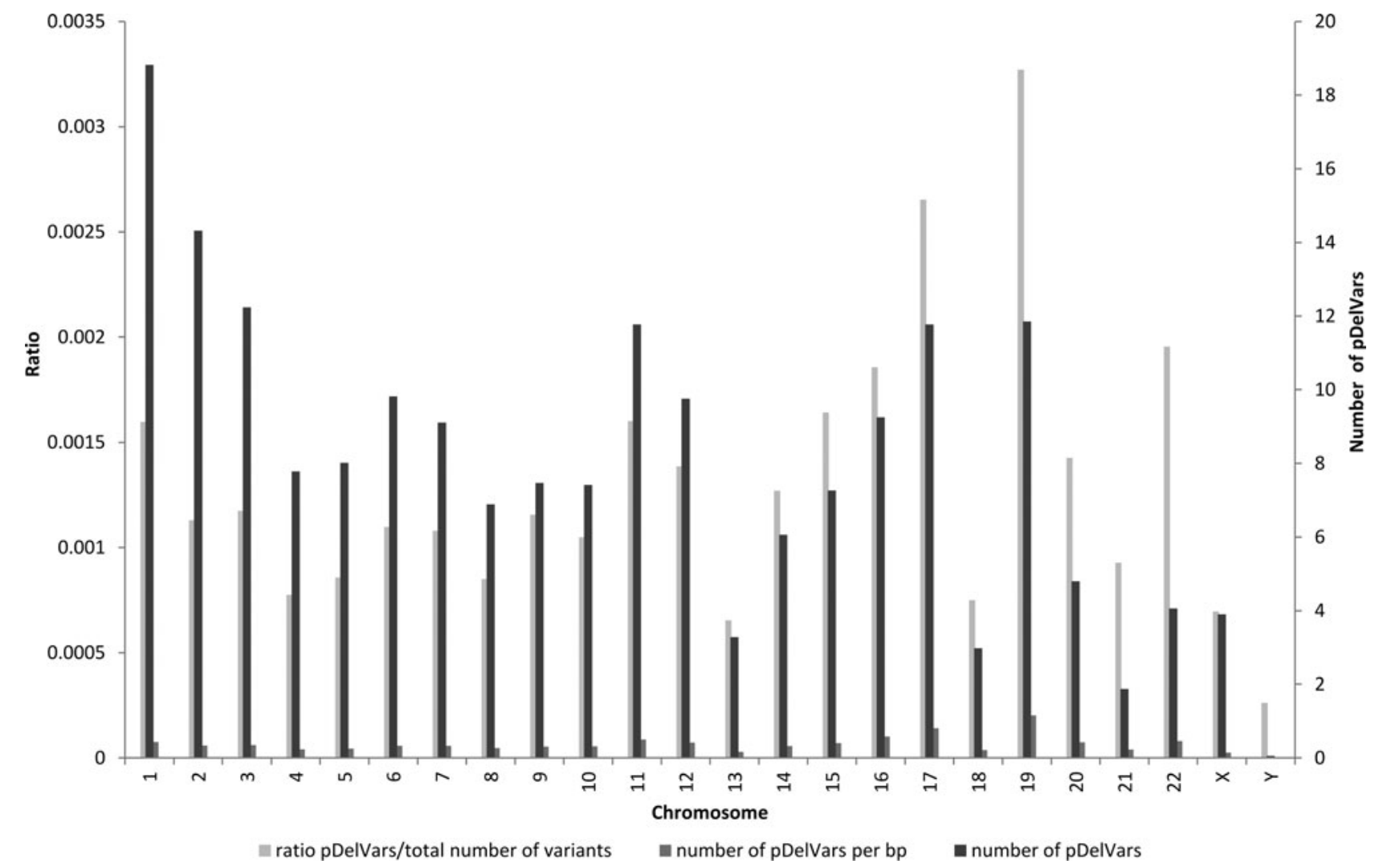

SUPPLEMENTARY FIG. S1. Statistics of pDelVars per chromosome in humans. Number of pDelVars per bp, ratio deleterious/total number of sequence variants according to chromosome, and absolute number of potentially deleterious sequence variants according to chromosomes. pDelVars, potentially deleterious variants. 


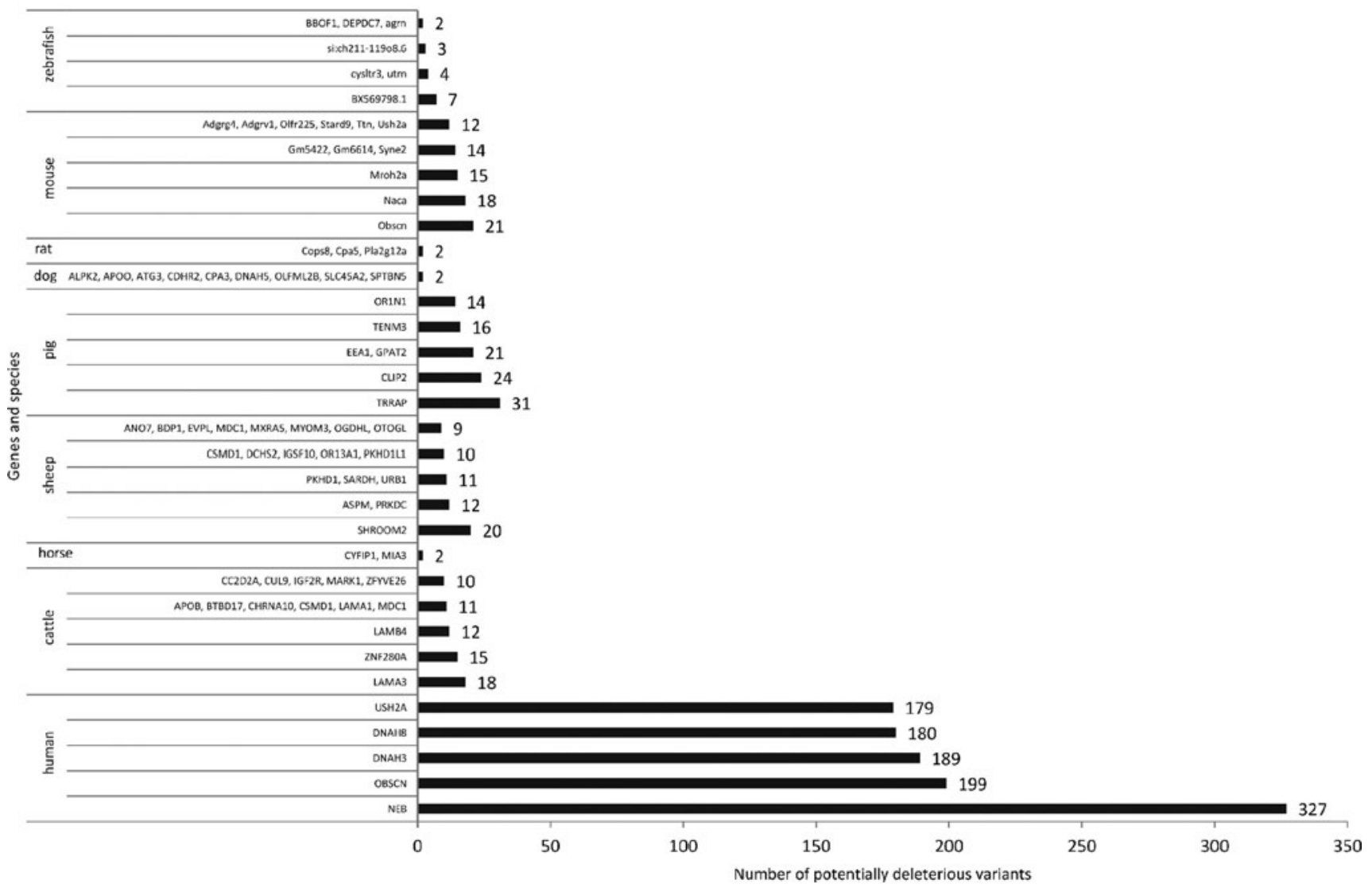

SUPPLEMENTARY FIG. S2. Genes with the highest number of pDelVars in genomes of nine vertebrate species. 
A

Human
Chimpanze
Gorilla
Orangutan
Vervet-2as:
Yacague
Olive baboos
Varmos:
Veuse
Ancestral sequence
Rat
Rabbit
Cour
Sheep
Pig
Dog
Cas
Yorse

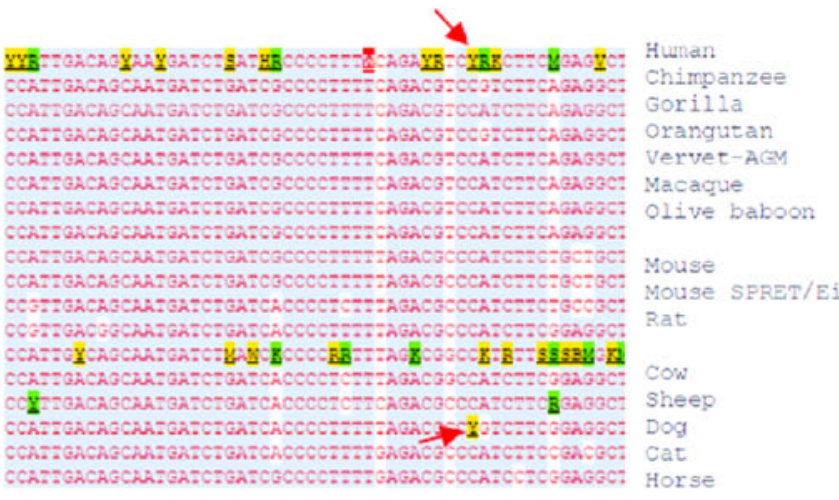

GACÄAGTGAGGCAATGAGCO GACAAGCTGAGGCAATGAGCE SACAAGCTGAGGCAATGAGC GACAAGCTCAGGCAATSAGC

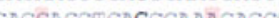
GCAAAGAGC GACGAGCTGAGGCAAAGAGCO GGAGACCGGCG G GCAAAGATCO GGAGACCGGCGGCAAAGATC: GGAGATATGCG $\rightarrow C A A A T A C C$ GATAA GCTGAG:C CAATGAGS Gampactomgachatsago

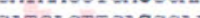
GATCAOTTGAGOCAAT GAGCO GATGAGCIGAGSCAATGAGC GATGAGTIGAGGCAAT GAGC:

B Human Chimpanzes Gorilla oxangutan. vervez-hase Kacagua Ollve baboon Narmose: Rabbit Rat Sheep Big lig Dog Horge Human Chimpanzee Gorilia Orangutan

Vervet-hov Wacaque Olive baboon Varmoset Rabbit Rat cow Sheep Pig

Dog Cat
Ho:se

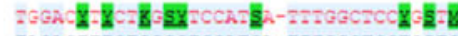



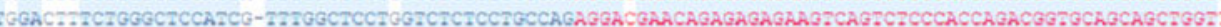

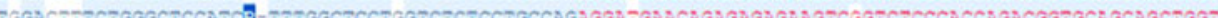

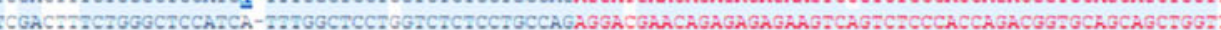

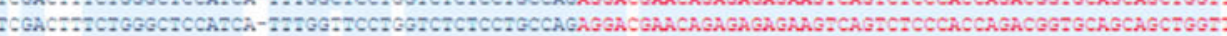

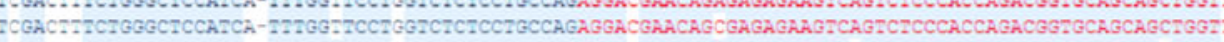

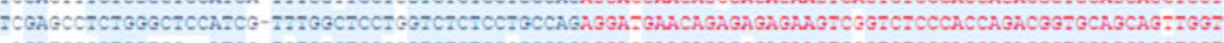

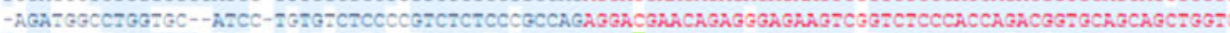


-

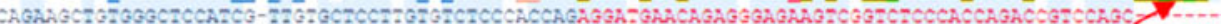



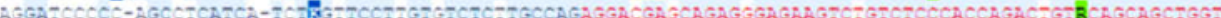

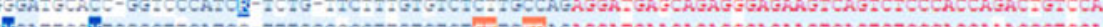

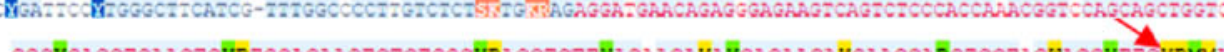

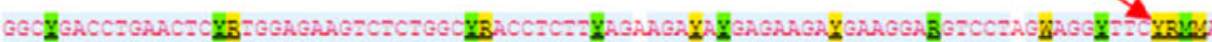

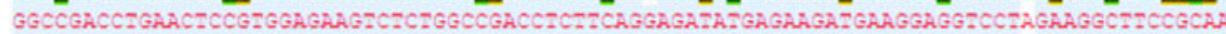
GGCCGACCTGAACTCCGTGGAGAAGTCRCTGGCIGACCTCZTCAGGAGATATGAGAAGATGAAGGAGGICCTAGAAGGCTICCGCAA GGCCGACCIGAACZCCGIGGAGAAGZCZCIGGCCGACCICZTCAGGAGATATGAGAAGATGAAGGAGGICCZAGAAGGCZTCCGAA

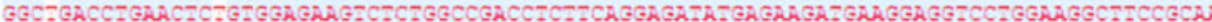

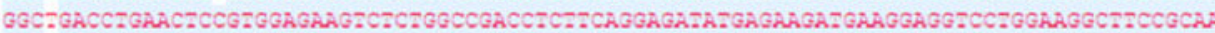

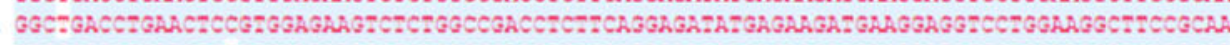
GGCCGACCZGAACZCZGIGGAGARGZCCTGGCCGACCZCZTCAGGAGAZATGAGAAGAGAAGGAGGZCCZGGAGGCZTCCGCA

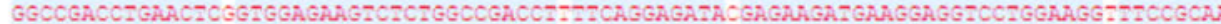

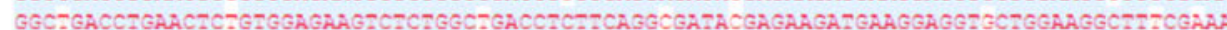

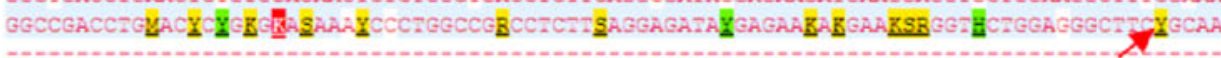

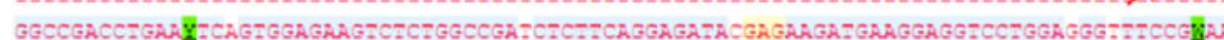

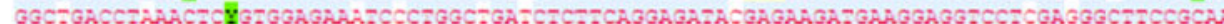



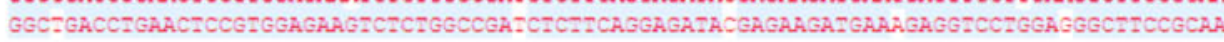

C

Cow

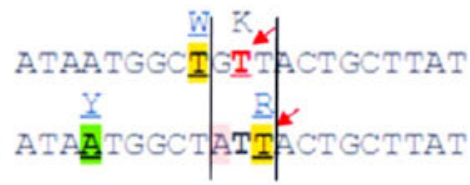

D Human Chimpanzee

Gorilla Orangutan vervet-AGM Macaque Olive baboon Marmoset

Mouse Mouse SPRETEIJ Rabbit Cow Sheep

Pig Dog Cat Horse

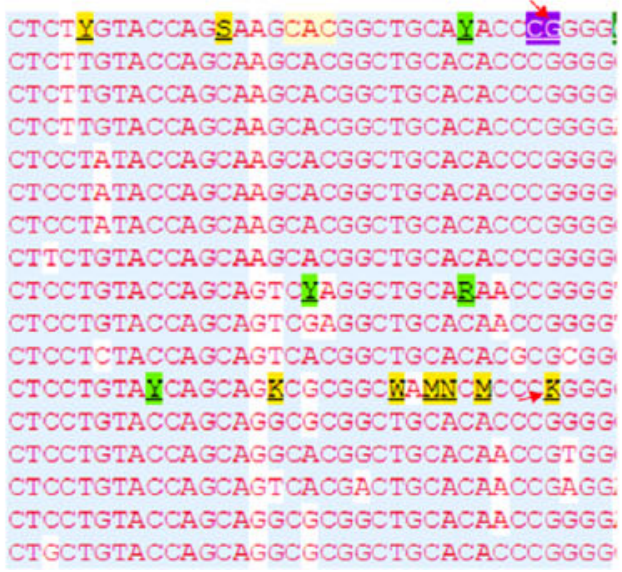

SUPPLEMENTARY FIG. S3. Multiple genomic alignments of the selected genes with marked orthologous (potentially deleterious) variants. (A) MPDZ gene: dog—rs22153300 and human-rs150393677; MPDZ gene: mouse-rs28101990 and human-rs779688439. (B) TACC2 gene: human-rs756574052 and cattle-rs455330779; human-rs761622230 and cattle-rs479667924. (C) TLR7 gene: human—rs34501186 and cattle-rs133254244 (codon marked with vertical lines). (D) RYRl gene: human—rs193922839 and cattle—rs474030293. 
Supplementary Table S1. Number of Potentially Deleterious Variants in Each Individual Chromosome in Human Genome

\begin{tabular}{|c|c|c|c|c|c|}
\hline Chromosome & $\begin{array}{c}\text { No. of } \\
\text { pDelVars }\end{array}$ & $\begin{array}{c}\text { No. of pDelVars } \\
\text { experimentally } \\
\text { validated }\end{array}$ & Chromosome & $\begin{array}{c}\text { No. of } \\
\text { pDelVars }\end{array}$ & $\begin{array}{c}\text { No. of pDelVars } \\
\text { experimentally } \\
\text { validated }\end{array}$ \\
\hline 1 & 18,824 & 17,290 & 14 & 6063 & 5633 \\
\hline 2 & 14,319 & 13,154 & 15 & 7261 & 6676 \\
\hline 3 & 12,234 & 11,230 & 16 & 9254 & 8608 \\
\hline 4 & 7782 & 7139 & 17 & 11,774 & 10,477 \\
\hline 5 & 8012 & 7406 & 18 & 2979 & 2732 \\
\hline 6 & 9820 & 9044 & 19 & 11,857 & 10,982 \\
\hline 7 & 9105 & 8440 & 20 & 4796 & 4414 \\
\hline 8 & 6889 & 6353 & 21 & 1872 & 1706 \\
\hline 9 & 7466 & 6940 & 22 & 4060 & 3772 \\
\hline 10 & 7416 & 6858 & $\mathrm{X}$ & 3901 & 3137 \\
\hline 11 & 11,771 & 10,839 & $\mathrm{Y}$ & 55 & 52 \\
\hline 12 & 9759 & 8880 & Total & 190,547 & 174,779 \\
\hline 13 & 3278 & 3017 & & & \\
\hline
\end{tabular}

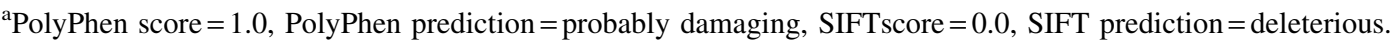
pDelVars, potentially deleterious variants; PolyPhen, polymorphism phenotyping; SIFT, sorts intolerant from tolerant. 


\section{AUTHOR QUERY FOR OMI-2018-0046-VER9-ROZMAN_1P}

AU1: Please note that gene symbols in any article should be formatted per the gene nomenclature. Thus, please make sure that gene symbols, if any in this article, are italicized.

AU2: Please review all authors' surnames for accurate indexing citations.

AU3: The Publisher requests for readability that all paragraphs be limited to 15 lines. Please adjust all paragraphs accordingly.

AU4: Ref. "Abecasis et al. (2011)" is cited in the text, but not found in the Ref. list. Please check.

AU5: Kindly check author name "Huang da W" is OK as given in Ref. "Jiao et al. (2012)."

AU6: In Ref. "Skrlj et al. (2017)," please mention the page range.

AU7: Please confirm corresponding author's address.

AU8: Acronym "PSI-BLAST" is not used in the article. Hence "PSI-BLAST" has been deleted from the abbreviations list.

AU9: Please mention the significance of "colors" in Table 3.

AU10: Please mention the significance of "arrows and different highlights" in Supplementary Fig. S3. 